\title{
Influence of the terrestrial magnetic field geometry on the cutoff rigidity of cosmic ray particles
}

\author{
K. Herbst, A. Kopp, and B. Heber \\ Institut für Experimentelle und Angewandte Physik, Christian-Albrechts-Universität zu Kiel, Kiel, Germany \\ Correspondence to: K. Herbst (herbst@ physik.uni-kiel.de)
}

Received: 23 May 2013 - Revised: 30 July 2013 - Accepted: 15 August 2013 - Published: 2 October 2013

\begin{abstract}
Studies of the propagation of charged energetic particles in the Earth's magnetic field go back to Carl Størmer. In the end, his investigations finally lead to the definition of the so-called cutoff rigidity $R_{\mathrm{C}}$; that is, the minimum momentum per charge a particle must have in order to reach a certain geographical location. Employing Monte Carlo simulations with the PLANETOCOSMICS code we investigate the correlation between the geomagnetic field structure and the cutoff rigidity. We show that the geometry of the magnetic field has a considerable influence on the resulting cutoff rigidity distribution. Furthermore, we will present a simple geometry-based parameter, $\delta B$, which is able to reflect the location-dependent cutoff rigidity. We show that this correlation is also visible in the temporal evolution of the Earth's magnetic field, at least over the last $100 \mathrm{yr}$. Using latitude scans with neutron monitors, changes of the relative counting rates at different positions are calculated, showing small variations for, e.g., Kiel and Moscow, while large ones occur at Mexico City as well as on the British Virgin Islands.
\end{abstract}

Keywords. Geomagnetism and Paleomagnetism (Time variations, secular and long term) - Interplanetary Physics (Cosmic rays) - Magnetospheric Physics (Energetic particles, precipitating)

\section{Introduction}

For particles, with momentum $p$ and charge $q$, interacting with the Earth's magnetic field $B$, the concept of the cutoff rigidity $R_{\mathrm{C}}$, where the rigidity $R$ is defined as $R=p / q=$ $r_{\mathrm{L}} \cdot B$, with $r_{\mathrm{L}}$ representing the Larmor radius, is used as a measure for the ability of a particle to penetrate the magnetic field at a certain location. In a geocentric dipole field the cutoff rigidity - that is, the minimum momentum per charge a particle must have in order to reach a certain location - can be described by the Størmer cutoff rigidity $R_{\mathrm{C}}$. Its approximation is given by

$$
\begin{aligned}
R_{\mathrm{C}} & =\kappa \frac{1}{L^{\alpha}} \\
& =\frac{M \cdot \cos ^{4} \lambda}{4 \cdot r^{2}},
\end{aligned}
$$

where $\kappa \approx 14.823 \mathrm{GV}, \alpha=2.0311$ and $L$ represents the McIlwain parameter, which indicates the distance at which a magnetic field line crosses the equatorial plane (see Shea and Smart, 1986; Pilchowski et al., 2010). Moreover, $M$ represents the dipole moment, $r$ the distance from the dipole center (in units of Earth radii) and $\lambda$ the geographic latitude (see, e.g., Shea and Smart, 1970, for further information) indicating that $R_{\mathrm{C}} \propto B$. Equation (2) is widely used, although it is, strictly speaking, only valid for high latitudes (see Pilchowski et al., 2010), and does not take into account the field geometry. In addition, the Earth's magnetic field configuration is much more complex than a simple dipole. Thus, in order to compute $R_{\mathrm{C}}$ in an arbitrary magnetic field, numerical computations are mandatory. For our studies we use the simulation code PLANETOCOSMICS (Desorgher, 2006). Information on the computation method is given in the Appendix.

On the basis of the investigations by Pilchowski et al. (2010) and Fichtner et al. (2012) the influence of the magnetic field geometry and its magnitude on the computed vertical cutoff rigidity is investigated in the following in more detail. Note that for simplicity the term cutoff rigidity in the following addresses the vertical cutoff rigidity. 


\section{Temporal evolution of the cutoff rigidity and its connection with the magnetic field geometry}

In order to investigate the correlation between the geomagnetic field structure and the cutoff rigidity, the magnitude of the magnetic field $|\boldsymbol{B}|$ as well as the measure $\delta B$, defined below, representing the field geometry will be compared with computations of the cutoff rigidity.

\subsection{Connection between cutoff rigidity and magnetic field geometry}

Although the Earth's magnetic field may be regarded as a tilted dipole field in a first-order approximation, C. F. Gauss noted in the 19th century that the field may better be represented by a series of Legendre polynomials. A commonly used model of the Earth's internal magnetic field, in particular for investigations between 1900 and today, is the International Geomagnetic Reference Field (IGRF; see, e.g., Finlay et al., 2010). The IGRF is a spherical harmonic model with coefficients derived from satellites and ground-based instruments for which every five years a new set of parameters is released by the International Association of Geomagnetism and Aeronomy (IAGA). However, for purpose of the present study, similar models like the POtsdam Magnetic Model of the Earth (POMME) 3 and 4, merely based on satellite data, are also applicable in the same way. Following the discussion in Pilchowski et al. (2010) as well as in Fichtner et al. (2012), we introduce the difference between the horizontal and vertical components as a measure for the geometry of the magnetic field given by

$\delta B=\sqrt{B_{\vartheta}^{2}+B_{\varphi}^{2}}-\left|B_{r}\right|$

in a spherical coordinate system. Thus, no further computations are needed in order to estimate $\delta B$. Figure 1 shows the distribution of magnitude and geometry measure (upper and middle panels) in comparison to the cutoff rigidity obtained with the PLANETOCOSMICS code (lower panel).

It becomes obvious that the cutoff rigidity values are lowest at polar regions, and reach amounts above $10 \mathrm{GV}$ close to the equator. The magnitude of the magnetic field $|\boldsymbol{B}|$, however, shows a lower order structure with islands of high values, especially south of Australia, as well as low value areas in particular between South America and southern Africa, known as the South Atlantic Anomaly (SAA), which cannot be seen in the values of the cutoff rigidity. In contrast to $|\boldsymbol{B}|$, the geometry of the field represented by $\delta B$ shows a behavior quite similar to $R_{\mathrm{C}}$. Despite the curved shape of the magnetic field, the significant maximum at equatorial regions can be found as well. Comparing $\delta B$ and $R_{\mathrm{C}}$, we also find a good agreement for latitudes greater then $50^{\circ}$, while at higher latitudes the importance of $|\boldsymbol{B}|$ becomes more noticeable. Thus, note that (a) $\delta B$ can only be seen as a first-order approximation because of the differences to $R_{\mathrm{C}}$ at, e.g., polar regions and that (b) residue features of $|\boldsymbol{B}|$ are still visible in $R_{\mathrm{C}}$.

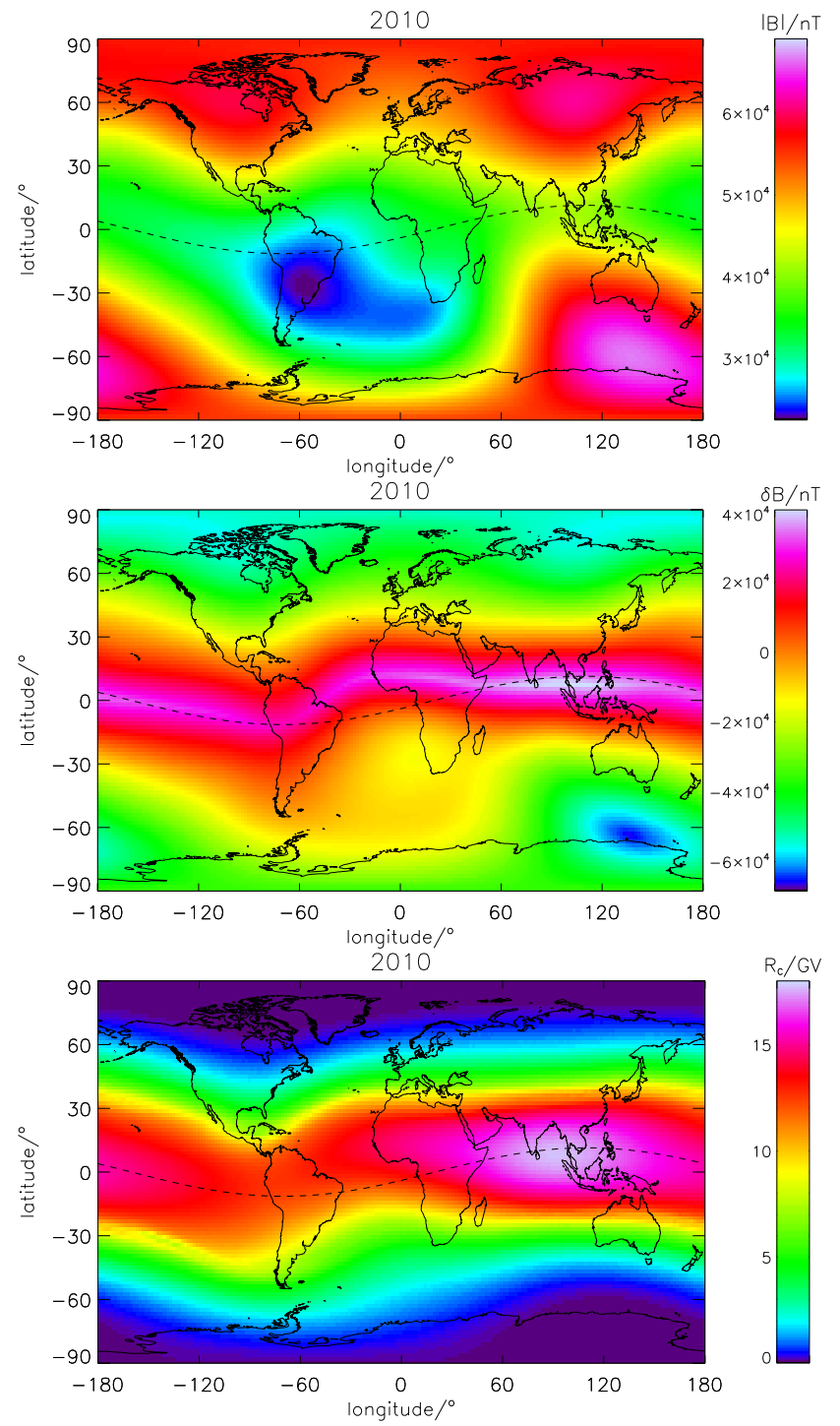

Fig. 1. The three parameters $|\boldsymbol{B}|$ (upper panel), $\delta B$ (middle panel) and $R_{\mathrm{C}}$ (lower panel) of the magnetic field configuration using the IGRF model for the year 2010.

This, however, clearly reveals that the vertical cutoff rigidity is much better reflected by the geometry of the magnetic field than by the magnitude $|\boldsymbol{B}|$.

In a second step we will study the temporal variation of $R_{\mathrm{C}}$, the magnitude $|\boldsymbol{B}|$ and $\delta B$ between 1900 and 2010 in order to investigate our findings in more detail .

\subsection{Temporal evolution of the magnetic field quantities}

To investigate the evolution of $|\boldsymbol{B}|$ (left panels), $\delta B$ (middle panels) as well as $R_{\mathrm{C}}$ (right panels), the global distributions for the year 1900 (upper panels), 1955 (middle panels) and 2010 (lower panels) are displayed in Fig. 2, showing the following: 

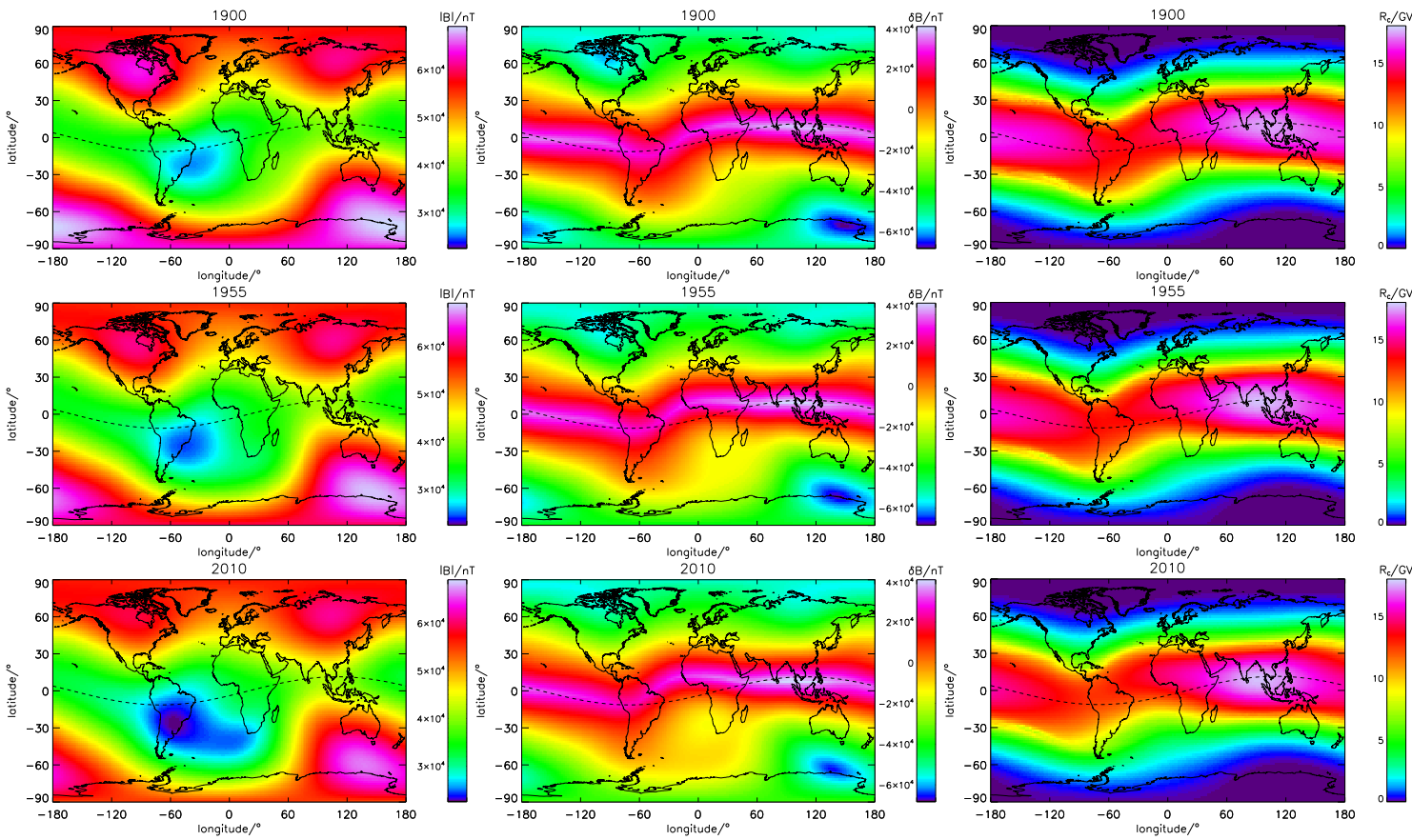

Fig. 2. The three parameters $|\boldsymbol{B}|$ (left panels), $\delta B$ (middle panels) and $R_{\mathrm{C}}$ (right panels) of the magnetic field configuration using the IGRF model for the years 1900 (upper panels), 1955 (middle panels) and 2010 (lower panels). A detailed description is given in the text.

a. The temporal variation of the cutoff rigidity $R_{\mathrm{C}}$ reveals a slight shift of the high-cutoff-rigidity band in northwestern direction, which particularly can be seen in the increase of the cutoff-rigidity values at the region between North America, North Africa and southern Europe. Additionally, an increase as well as a longitudinal widening of the maximum values can be observed.

b. The quantity $\delta B$ follows the temporal evolution of the cutoff rigidity, i.e., the westward motion of the high value band. In contrast, however, also stronger temporal variations at polar regions can be observed.

c. The magnitude of the magnetic field also shows significant variations, which, however, again do not show obvious correlations with $R_{\mathrm{C}}$. The most prominent effects are a significant decrease of the magnetic field intensity in the SAA as well as a longitudinal and latitudinal extension of this area.

As a consequence of the latter point, the temporal variations of $|\boldsymbol{B}|$ will not be studied further.

Figure 3 displays the absolute differences of $R_{\mathrm{C}}$ and $\delta B$ between the years 1900 and 2010. It shows that the cutoff rigidity (upper panel) has significantly changed within the last century, especially over central Europe, Asia, Australia and South Africa, while only small changes can be observed at high latitudes. In particular, three regions of significant variations well beyond $\pm 3 \mathrm{GV}$ can be found: the region over Mexico City, the region between South America and South

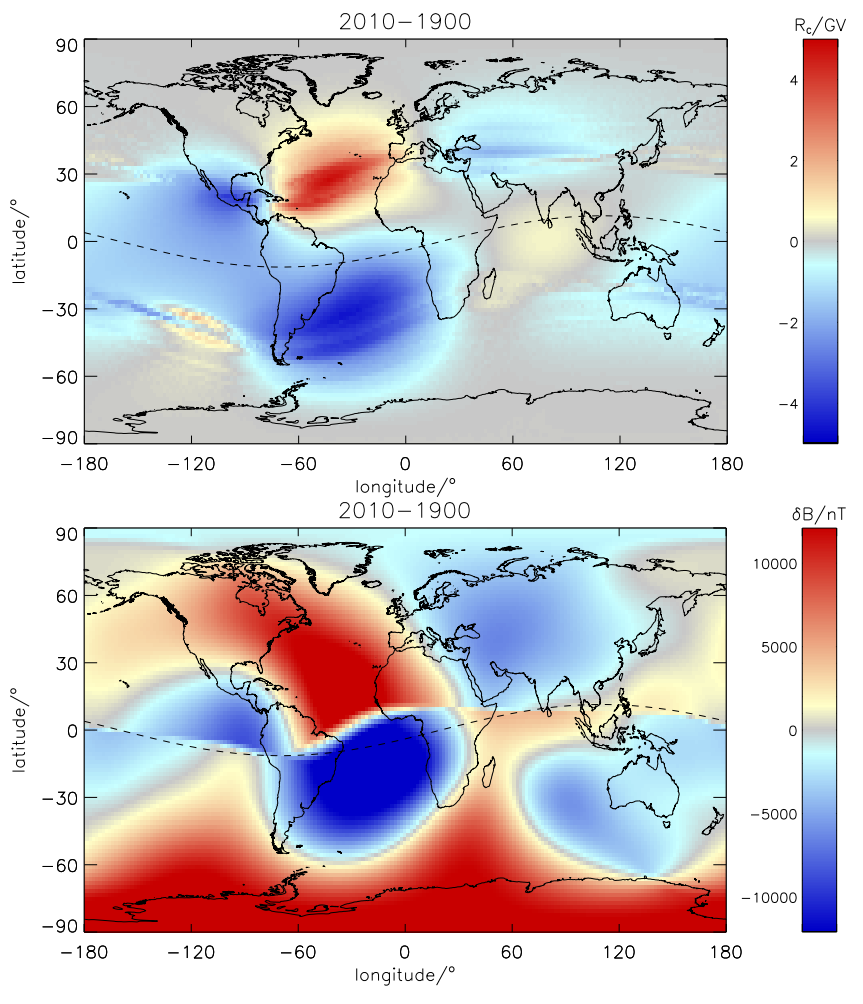

Fig. 3. The absolute differences of $R_{\mathrm{C}}$ (upper panel) and $\delta B$ (lower panel) between 1900 and 2010. 

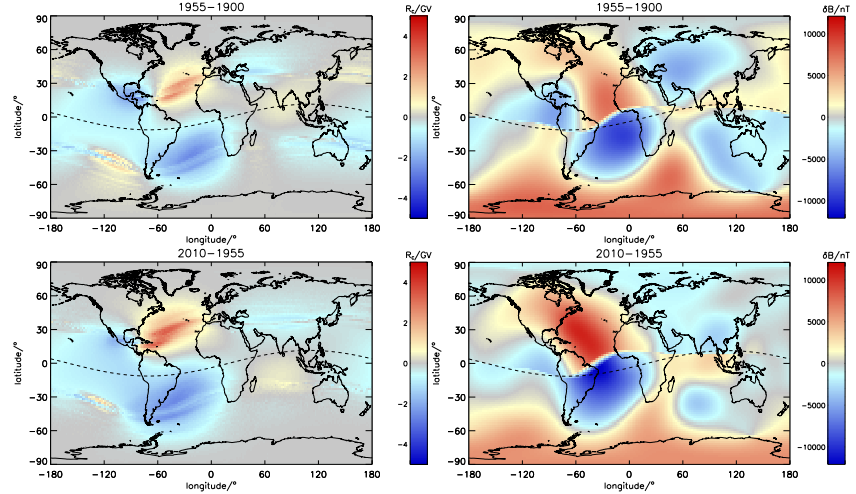

Fig. 4. Same as Fig. 3 but for the intervals 1900 to 1955 (upper panels) and 1955 to 2010 (lower panels)

Africa (decrease), and the region between North-America, North Africa and southern Europe (increase). Similar behavior can be found at low and midlatitudes of the $\delta B$ values (lower panel), while at high latitudes again significant differences are observed. The regions of strong decreases as well as increases, however, correlate with those occurring in the changes of the cutoff rigidity.

Another important fact is that the geomagnetic field underwent much stronger changes between 1955 and 2010 than in the years between 1900 and 1955, as displayed in the left panels of Fig. 4. Additionally, the corresponding $\delta B$ variations are shown in the right panels, revealing again a quite similar behavior.

\section{Influence of the temporal variations of $R_{C}$ on ground-based measurements}

With the invention of the neutron monitor (NM; see, e.g., Simpson, 2000), it became possible to measure the altitudeand latitude-dependent cosmic-ray flux at the Earth's surface. Especially the temporal evolution of $R_{\mathrm{C}}$ should have an effect on the secondary particle environment, the particles being produced by the interaction of cosmic rays with the Earth's atmosphere, and thus should be visible in NM data. Therefore, the influence of the temporal cutoff rigidity variations at selected locations are investigated in the following.

The green-filled stars in Fig. 5 show the NM stations that have been active since 1955. The red and blue ellipses mark the areas of significant decreases as well as increases of $R_{\mathrm{C}}$, within which only a few NM stations are located.

In order to investigate the influence of the rigidity changes on the NM counting rates, four specific locations (colored in magenta) are investigated: Kiel $\left(54^{\circ} \mathrm{N}, 11^{\circ} \mathrm{E}\right)$, Moscow $\left(56^{\circ} \mathrm{N}, 38^{\circ} \mathrm{E}\right)$, Mexico City $\left(19^{\circ} \mathrm{N}, 100^{\circ} \mathrm{W}\right.$, within the blue circle) and a hypothetical NM on the British Virgin Islands $\left(18^{\circ} \mathrm{N}, 64^{\circ} \mathrm{W}\right.$, within the red circle, abbreviated as BVI).

The panels of Fig. 6 show the temporal variations of the cutoff rigidity (left panels) as well as the geometry measure

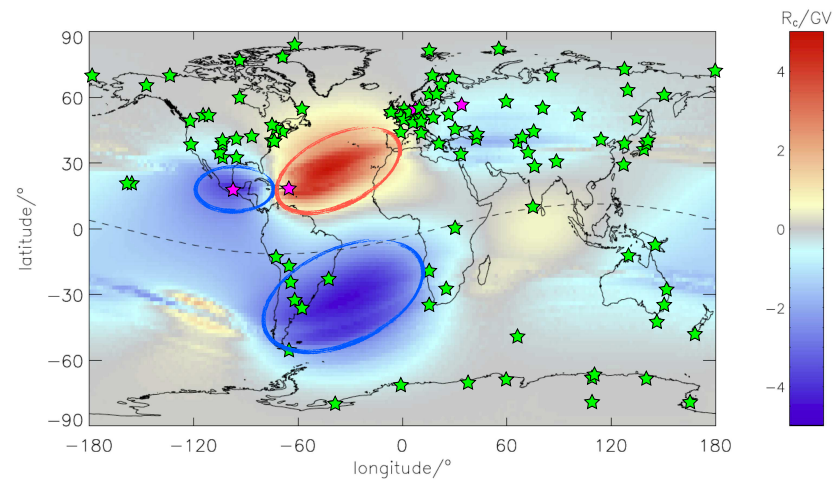

Fig. 5. Neutron monitor stations established between 1950 and 2012 (filled green stars). Colored in magenta are the stations used for our study: Kiel, Moscow, Mexico City and the hypothetical station at the British Virgin Islands

$\delta B$ (right panels) for Moscow and Kiel (given as red and black dots, respectively) in the upper panels, while Mexico City (black dots) and BVI (red dots) are displayed in the lower panels. Reviewing this information, the following becomes obvious:

a. Although Kiel and Moscow are located at nearly equal latitudes, strong differences in the temporal evolution of both values, the cutoff rigidity values (upper left panel) and the $\delta B$ values (upper right panel) between 1900 and 2010 can be observed. While at Kiel (black dots) only minor variations are visible, Moscow (red dots) shows clearly visible decreases of both measures.

b. Considerably stronger temporal variations become obvious at Mexico City (lower left panel, black dots) and the British Virgin Islands (red dots).

c. The temporal evolution of $R_{\mathrm{C}}$ and $\delta B$ for all locations investigated here are in good agreement with each other. Note furthermore that in spite of the deviations at high latitudes, where, however, none of the investigated stations are located, the temporal variations of $\delta B$ are nevertheless in reliable agreement with the rigidity values, showing even similarities in the temporal behavior.

Thus, $\delta B$ is able to reflect also the temporal evolution of the Earth's magnetic field. For a better visualization the left panels also show color-coded lines referring to the years 1913 (blue), 1955 (magenta), 1976 (red) and 1997 (green) and their corresponding cutoff rigidity values, which are the basis of the investigations in Sect. 3. In addition, different line styles represent different locations. The solid lines in the upper (lower) left panel give the cutoff rigidity values of Kiel (Mexico City), while the dashed curves display the values of Moscow (British Virgin Islands). Unfortunately, only two of the used NMs are able to provide continuous data coverage 

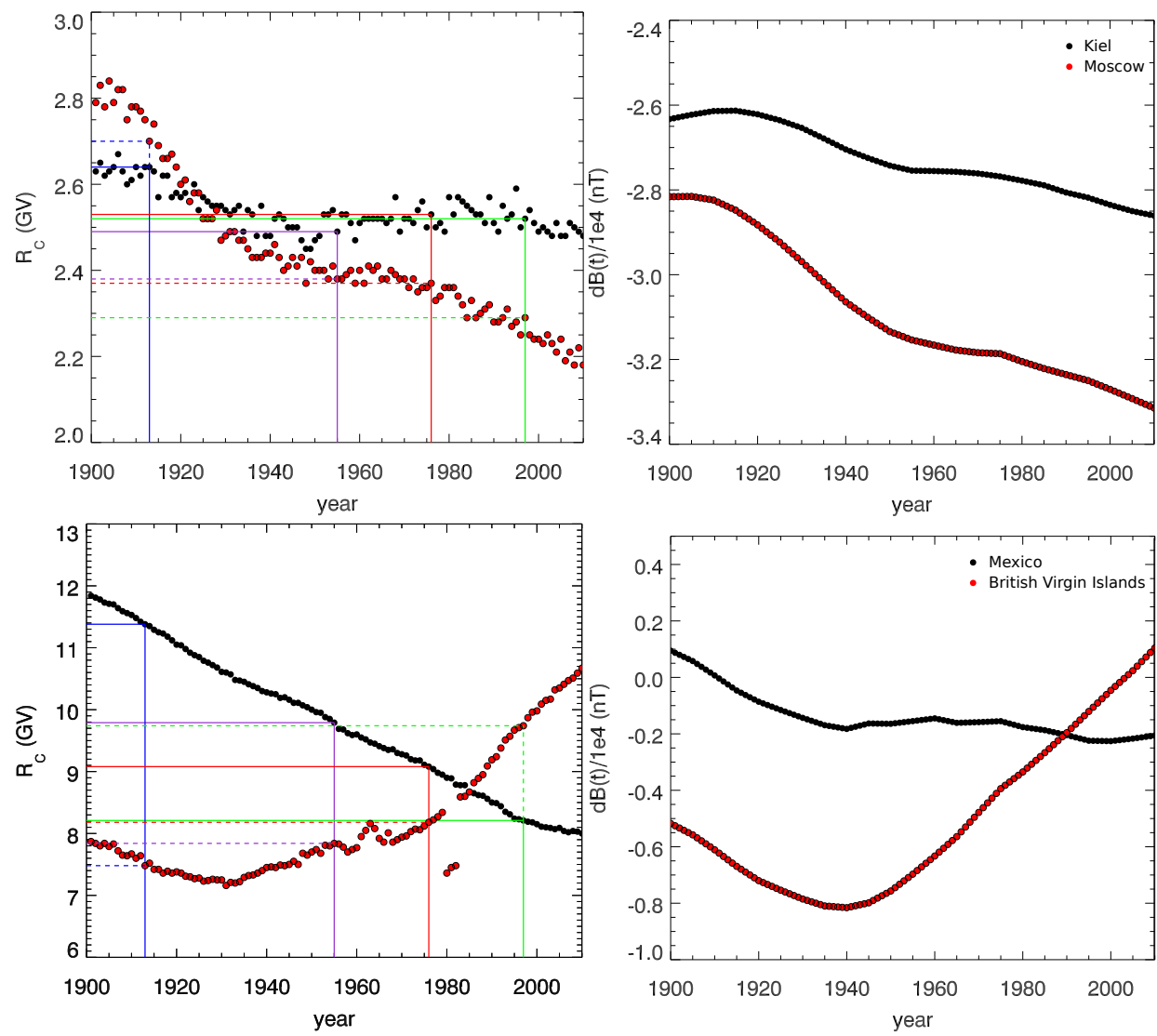

Fig. 6. Vertical cutoff rigidities (left panels) as well as $\delta B$ values (right panels) for the time interval between 1900 and 2010 at Kiel (black dots) and Moscow (red dots) in the upper panels as well as Mexico City (black dots) and the British Virgin Islands (red dots) displayed in the lower panels. The colored lines represent the years 1913 (blue), 1955 (dark orchid), 1976 (red) and 1997 (green), with solid (dashed) lines for Kiel and Mexico City (Moscow and BVI).

over decades, while the NM counting rates of, e.g., Mexico City cannot be used for a proper analysis over the given period of time.

However, another possibility to investigate the influence of the rigidity changes on the measured counting rates, and thus on the transport of cosmic ray particles, at certain locations is the analysis of NM latitude surveys (see, e.g., Clem and Dorman, 2000). In such surveys a mobile NM is used to measure the counting rates while covering several geomagnetic locations, and thus geomagnetic cutoffs (see Moraal et al., 1989). Assuming the existence of a correlation between cutoff rigidities and measured counting rates, but being aware of the fact that this assumption has its limitations (e.g., in the SAA; see Fichtner et al., 2012), we use the Italian Antarctic Program 3-NM-64 survey (see Villoresi et al., 1997) for our analysis. This survey was performed during the solar minimum conditions of 1997 with a modulation value $\phi$ of $410 \mathrm{MV}$ (see Usoskin et al., 2011).

To investigate the influence of the temporal cutoff rigidity changes presented in the previous section, it is of great importance to only compare times with similar modulation values because the cosmic ray flux, and thus the counting rates measured during such surveys, strongly depend on the solar activity. According to Usoskin et al. (2011) an annual mean value of $\phi=410 \mathrm{MV}$ solely occurred during $A>0$ solar minimum conditions (see, e.g., Jokipii et al., 1977; Potgieter and Ferreira, 2001, for further information). Due to the magnetic solar cycle, these conditions occur about every $22 \mathrm{yr}$ (see also Usoskin et al., 2011, for comparison of the $\phi$ values). Thus, in the following the counting rate values of the four stations are investigated for the solar minimum conditions of 1997, 1976 and 1955. In addition, we also add a fourth time period to the investigations by extrapolating the lower edge of the available IGRF coefficients: 1913. The corresponding time- and location-dependent computed cutoff rigidity values are given in Table 1 .

By combining the rigidity computations with the results of the latitude survey, and thus, for each location-dependent cutoff rigidity value, a counting rate value can be determined. The procedure is displayed in Fig. 7. Here, each panel shows the measured counting rates normalized to the value at polar regions $\left(R_{\mathrm{C}}=0 \mathrm{GV}\right)$ as a function of the local cutoff rigidity 

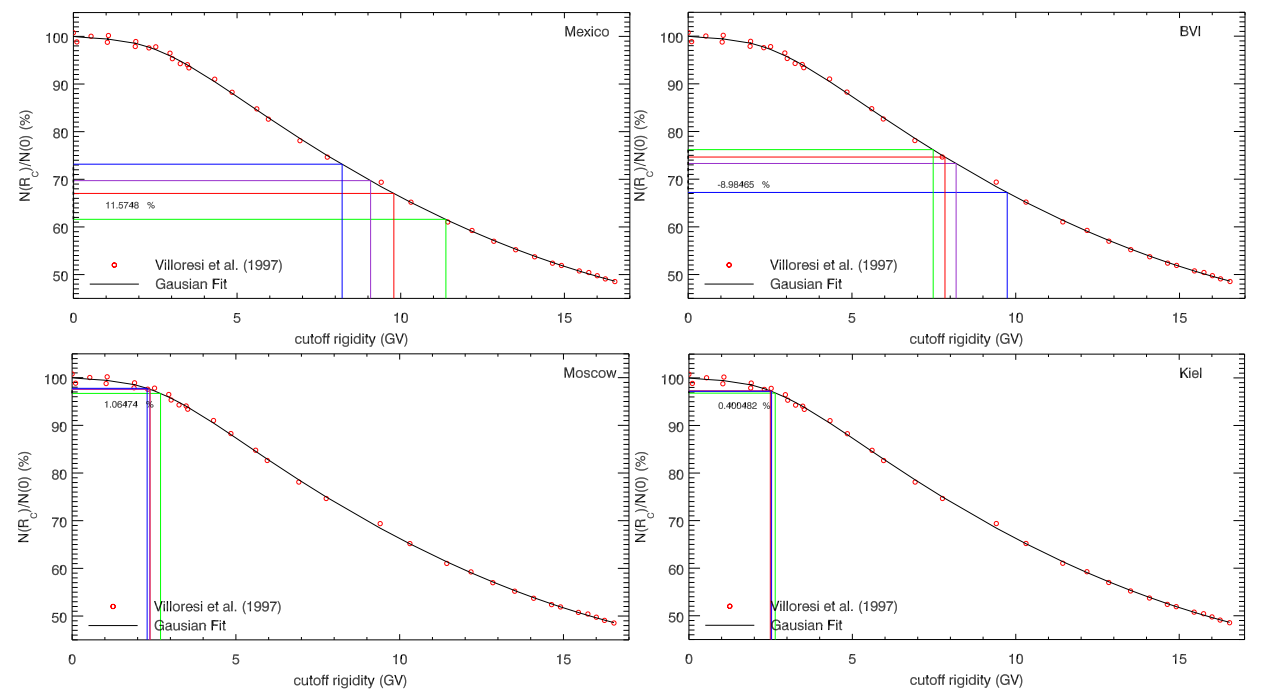

Fig. 7. NM latitude scan by Villoresi et al. (1997) (red circles, with a Gaussian fit plotted as black line) and the corresponding counting rate variations $N\left(R_{\mathrm{C}}\right)$ normalized to the counting rates at polar regions $N(0)$ at Mexico City, the BVIs, Moscow and Kiel. The color coding of the lines stand for the same years as in Fig. 6.

Table 1. Location-dependent computed cutoff rigidity values for times with an annual mean solar modulation value of $\phi=410 \mathrm{MV}$ (1913, 1955, 1976 and 1997; see Usoskin et al., 2011).

\begin{tabular}{rrrrr}
\hline Year & $\begin{array}{r}R_{\mathrm{C}, \text { Kiel }} \\
{[\mathrm{GV}]}\end{array}$ & $\begin{array}{r}R_{\mathrm{C}, \text { Moscow }} \\
{[\mathrm{GV}]}\end{array}$ & $\begin{array}{r}R_{\mathrm{C}, \text { Mexico }} \\
{[\mathrm{GV}]}\end{array}$ & $\begin{array}{r}R_{\mathrm{C}, \mathrm{BVI}} \\
{[\mathrm{GV}]}\end{array}$ \\
\hline 1913 & 2.64 & 2.70 & 11.38 & 7.48 \\
1955 & 2.49 & 2.38 & 9.79 & 7.84 \\
1976 & 2.53 & 2.37 & 9.08 & 8.18 \\
1997 & 2.52 & 2.29 & 8.21 & 9.74 \\
\hline
\end{tabular}

(red circles). To interpolate over the entire cutoff rigidity range, in addition, a Gaussian profile has been fitted to the measurements (black solid line). Using the computed cutoff rigidity values (given in Table 1), we are thus able to read out four time-dependent normalized counting rate values for each location. Each year is color coded as given above. An overview of the actual location-dependent $N\left(R_{\mathrm{C}}\right) / N(0)$ values is given in Table 2.

Assuming the normalized counting rates as a function of $R_{\mathrm{C}}$ to be the same over the investigated times, it becomes obvious that an increase of about $11.6 \%$ at Mexico City (upper left panel) between 1997 and 1913 occurs, while a decrease of about $9 \%$ is revealed on the British Virgin Islands (upper right panel). Stations with almost no temporal cutoff decreases and increases (e.g., Kiel and Moscow displayed in the lower panels), however, show weaker counting rate variations. At these locations the minor variations cannot be distinguished from the statistical noise of the detector, and thus can be neglected. However, note that the numbers also have to be put in contrast to the overall solar cycle variations at the different neutron monitor stations.
Table 2. Read-out counting rates during the solar minimum conditions of 1913, 1955, 1976 and 1997.

\begin{tabular}{rrrrr}
\hline & $N\left(R_{\mathrm{C}}\right) /$ & $N\left(R_{\mathrm{C}}\right) /$ & $N\left(R_{\mathrm{C}}\right) /$ & $N\left(R_{\mathrm{C}}\right) /$ \\
Year & $\begin{array}{r}N(0)_{\text {Kiel }} \\
{[\%]}\end{array}$ & $\begin{array}{r}N(0)_{\text {Moscow }} \\
{[\%]}\end{array}$ & $\begin{array}{r}N(0)_{\text {Mexico }} \\
{[\%]}\end{array}$ & $\begin{array}{r}N(0)_{\mathrm{BVI}} \\
{[\%]}\end{array}$ \\
\hline 1913 & 96.80 & 96.70 & 61.60 & 76.20 \\
1955 & 97.28 & 97.55 & 67.03 & 74.64 \\
1976 & 97.17 & 97.57 & 69.72 & 73.29 \\
1997 & 97.20 & 97.76 & 73.17 & 67.22 \\
\hline
\end{tabular}

\section{Summary and conclusions}

The widely used formula by Shea and Smart (1970) (see Eq. 2), describing the vertical cutoff rigidity at a given location on Earth, depends only on the magnitude of the magnetic field strength $B$ and not on the geometry of the magnetic field. However, our previous investigations (see Pilchowski et al., 2010; Fichtner et al., 2012) showed the formula to be a too rough approximation, and suggested the cutoff rigidity to be related to the geometry of the magnetic field. Following this idea we investigated the influence of the geometry on the magnetic field on the vertical cutoff rigidity $R_{\mathrm{C}}$.

We could find a quantity $\delta B$, representing the difference between the horizontal and vertical components of the magnetic field, which suitably well reflects the vertical cutoff rigidity beyond the polar regions. Moreover, we could show that this geometry measure is able to reflect the temporal evolution of $R_{\mathrm{C}}$, revealing the existence of regions with strong geometry changes over the last $100 \mathrm{yr}$ that are strong enough to be measurable by neutron monitors. We conclude that the geometry of the magnetic field, in contrast to the previous 
assumption, has a non-negligible influence on the (vertical) cutoff rigidity. However, information on $|\boldsymbol{B}|$ in addition to $\delta B$ is required in order to understand the cutoff rigidity $R_{\mathrm{C}}$.

\section{Appendix A}

\section{On the computation method}

PLANETOCOSMICS, developed by Laurent Desorgher at the University of Bern (Switzerland), provides the opportunity to compute the transport of charged particles within the Earth's magnetosphere as well as their interaction with the Earth's atmosphere. The base for computing the cutoff rigidity distribution is Størmers transport equation for charged particles (see Størmer, 1955). Backward trajectories are thereby simulated for a set of rigidity values spanning over a wide range. From these computations mainly three rigidity regions can be identified: (i) the allowed region, where all trajectories are allowed; (ii) the forbidden region, where all trajectories are forbidden; and (iii) the penumbral region, where bands of allowed trajectories are interjected by bands of forbidden ones. Thus, the rigidity of the last allowed computed rigidity before the first forbidden one is known as the upper cutoff rigidity $R_{\mathrm{U}}$, while the rigidity of the last allowed trajectory, below which all other trajectories are forbidden, is called the lower cutoff rigidity $R_{\mathrm{L}}$. The effective cutoff rigidity $R_{\mathrm{C}}$ then can be approximated by

$R_{\mathrm{C}}=R_{U}-\sum_{i=R_{\mathrm{L}}}^{R_{\mathrm{U}}} \Delta R_{i} \quad$ (allowed).

A complete and more detailed description of this method can be found in Cooke et al. (1991).

Acknowledgements. We are grateful for support for the research by the Deutsche Forschungsgemeinschaft (DFG) via the project "Heliocauses" (HE 3279/8-2 and HE 3279/8-3) carried out within the framework of the DFG priority program 1176 within CAWSES.

Topical Editor I. Daglis thanks two anonymous referees for their help in evaluating this paper.

\section{References}

Clem, J. M. and Dorman, L. I.: Neutron monitor response functions, Space Sci. Rev., 93, 335-359, 2000.

Cooke, D. J., Humble, J. E., Shea, M. A., Smart, D. F., and Lund, N.: On cosmic-ray cut-off terminology, Nuovo Cimento C Geophys. Space Phys. C, 14, 213-234, 1991.

Desorgher, L.: The PLANETOCOSMICS code, available at: http://cosray.unibe.ch/ laurent/planetocosmics (last access: 26 September 2013), 2006.

Fichtner, H., Heber, B., Herbst, K., Kopp, A., and Scherer, K.: Solar Activity, the Heliosphere, Cosmic Rays and Their Impact on the Earth's Atmosphere, in: Climate And Weather of the SunEarth System (CAWSES): Highlights from a priority program, Springer, edited by: Lübken, F.-J., Dordrecht, the Netherlands, 55-78, 2012.

Finlay, C. C. and the Working Group V-MOD: International Geomagnetic Reference Field: the eleventh generation, Geophys. J. Int., 183, 1216-1230, 2010.

Jokipii, J. R., Levy, E. H., and Hubbard, W. B.: Effects of particle drift on cosmic-ray transport, I. General properties, application to solar modulation, Astrophys. J., 213, 861-868, 1977.

Moraal, H., Potgieter, M. S., Stoker, P. H., and van der Walt, A. J.: Neutron Monitor Latitude Survey of Cosmic Ray Intensity During the 1986/1987 Solar Minimum, J. Geophys. Res., 94, 14591464, 1989.

Pilchowski, J., Kopp, A., Herbst, K., and Heber, B.: On the definition and calculation of a generalised McIlwain parameter, Astrophys. Space Sci. T., 6, 9-17, 2010.

Potgieter, M. S. and Ferreira, S. E. S.: Modulation of cosmic rays in the heliosphere over 11 and 22 year cycles: a modelling perspective, Adv. Space Res., 27, 481-492, 2001.

Shea, M. A. and Smart, D. F.: On the application of trajectoryderived cutoff rigidities to cosmic ray intensity variations, Acta Phys., 29, 533-537, 1970.

Shea, M. A. and Smart, D. F.: Estimating cosmic ray vertical cutoff rigidities as a function of the McIlwain L-parameter for different epochs of the geomagnetic field, Phys. Earth Planet. Int., 48, 200-205, 1986.

Simpson, J. A.: The Cosmic Ray Nucleonic Component: The Invention And Scientific Uses Of Neutron Monitor, Space Sci. Rev. 93, 1-20, 2000.

Størmer, C.: The Polar Aurora, Clarendon Press, Oxford, 1955.

Usoskin, I. G., Brazlevskaia, G. A., and Kovaltsov, G. A.: Solar modulation parameter for cosmic rays since 1936 reconstructed from ground-based neutron monitors and ionization chambers, J. Geophys. Res., 116, A02104, doi:10.1029/2010JA016105, 2011.

Villoresi, G., Iucci, N., Tyasto, M. I., Dorman, L. I., Re, F., Signoretti, F., Zangrilla, N., Cecchini, S., Parisi, M., Signorini, C., Danilova, O. A., and Ptitsyna, N. G.: Latitude Survey of Cosmic Ray Nucleonic Component (Italy-Antarctic, 1996-1997), Proc. 25th Int. Cosmic Ray Conf., Durban, 2, no. 421, 1997. 